\title{
Interplay of superconductivity and magnetism in strong coupling
}

\author{
C. N. A. van Duin and J. Zaanen \\ Institute Lorentz for Theoretical Physics, Leiden University, P.O.B. 9506, 2300 RA Leiden, The Netherlands
}

(Received 17 August 1999)

\begin{abstract}
A model is introduced describing the interplay between superconductivity and spin ordering. It is characterized by on-site repulsive electron-electron interactions, causing antiferromagnetism, and nearest-neighbor attractive interactions, giving rise to $d$-wave superconductivity. Due to a special choice for the lattice, this model has a strong-coupling limit where the superconductivity can be described by a bosonic theory, similar to the strongly coupled negative $U$ Hubbard model. This limit is analyzed in the present paper. A rich mean-field phase diagram is found and the leading quantum corrections to the mean-field results are calculated. The first-order line between the antiferromagnetic and the superconducting phase is found to terminate at a tricritical point, where two second-order lines originate. At these lines, the system undergoes a transition to and from a phase exhibiting both antiferromagnetic order and superconductivity. At finite temperatures above the spin-disordering line, quantum-critical behavior is found. For specific values of the model parameters, it is possible to obtain $\mathrm{SO}(5)$ symmetry involving the spin and the phase sector at the tricritical point. Although this symmetry is explicitly broken by the projection to the lower Hubbard band, it survives on the mean-field level, and modes related to a spontaneously broken $\mathrm{SO}(5)$ symmetry are present on the level of the random phase approximation in the superconducting phase.
\end{abstract}

\section{INTRODUCTION}

Both for empirical and historical reasons, research on superconductivity tends to be preoccupied with the weak coupling limit. From a more general perspective, BCS theory as well as Gorkov-Migdal-Eliashberg theory correspond with a special case which in a sense is pathological. The emphasis is completely on the amplitude of the order parameter while fundamentally superconductivity is about breaking of gauge symmetry, associated with the phase sector. The work of Schmitt-Rink and Nozieres ${ }^{1}$ revealed that the BCS theory for a $s$-wave superconductor can be smoothly continued to the strong coupling limit. It is generally recognized that it is far easier to understand the vacuum structure of such a superconductor in strong coupling. Amplitude fluctuations can be regarded as highly massive excitations and all what remains is the phase sector described in terms of hardcore bosons, or alternatively in terms of pseudospin models.

In the context of high- $T_{c}$ superconductivity one encounters a far more complex physics. Abundant evidence is available for a $d$-wave superconducting order parameter. This is usually discussed in terms of weak-coupling theory with its $d$-wave nodal fermions while the more sophisticated approaches start from this limit, attempting to penetrate the intermediate coupling regime using self-consistent perturbation theory. ${ }^{2}$ The obvious problem is that the coherence length is rather short. ${ }^{3}$ At the same time, an interesting case has been presented claiming that much of the thermodynamics can be understood from phase dynamics alone, ${ }^{4}$ completely disregarding amplitude fluctuations. It would therefore be useful to study strong coupling theories for $d$-wave superconductors.

An even better reason to pursue a strong coupling perspective is the growing evidence for the presence of well developed antiferromagnetism coexisting with the superconductivity. Traditionally, this was approached within, again, an implicitly weakly coupled perspective. The magnetic fluctuations as seen in NMR and neutron scattering were believed to be due to the proximity to an amplitude driven spin density wave transition. ${ }^{5}$ Recently, this perspective has been drastically changed due to the observation of strong static antiferromagnetic order associated with the stripe phases in the $\mathrm{La}_{2} \mathrm{CuO}_{4}$ system. $^{6}$ In the $\mathrm{Nd}$ doped samples where this order is strongest the magnitude of the Néel order parameter can be as large as $0.3 \mu_{B},{ }^{7}$ while $0.1 \mu_{B}$ has been claimed in " "pristine", $\mathrm{La}_{1.88} \mathrm{Sr}_{0.12} \mathrm{CuO}_{4}{ }^{8}$ It appears that this antiferromagnetic order is in competition or even coexisting with the superconducting order. ${ }^{8,9}$ Given that the stripe antiferromagnet should be strongly renormalized downward due to transversal quantum spin fluctuations ${ }^{10}$ the stripe antiferromagnet has to be strongly coupled. Given the strong similarities between the static order and the incommensurate spin fluctuations which seem to be generic for all cuprate superconductors in the underdoped regime, a strong coupling perspective on the antiferromagnetism should be closer to the truth even if static order is not present, at least as long as the doping is not too large.

Recently several theoretical attempts have been undertaken to shed light on this problem of strongly coupled superconductivity and antiferromagnetism. The simplest theory of this kind is Zhang's $\mathrm{SO}(5)$ theory, where superconductivity and antiferromagnetism are "unified" within a single larger symmetry. ${ }^{11}$ Given that no such symmetry is manifestly present at the ultraviolet of the problem, this might well be misleading and one would like to have a more general framework in which this (near) $\mathrm{SO}(5)$ symmetry appears as a special case. The manifest symmetry of the problem is $\mathrm{U}(1) \times \mathrm{SU}(2)$ (superconducting phase- and spin, respectively). The structure of the long wavelength effective theory based on this symmetry principle has been analyzed recently by one of the authors, ${ }^{12}$ including the charge order associated with the stripe phase. These approaches are only truly mean- 


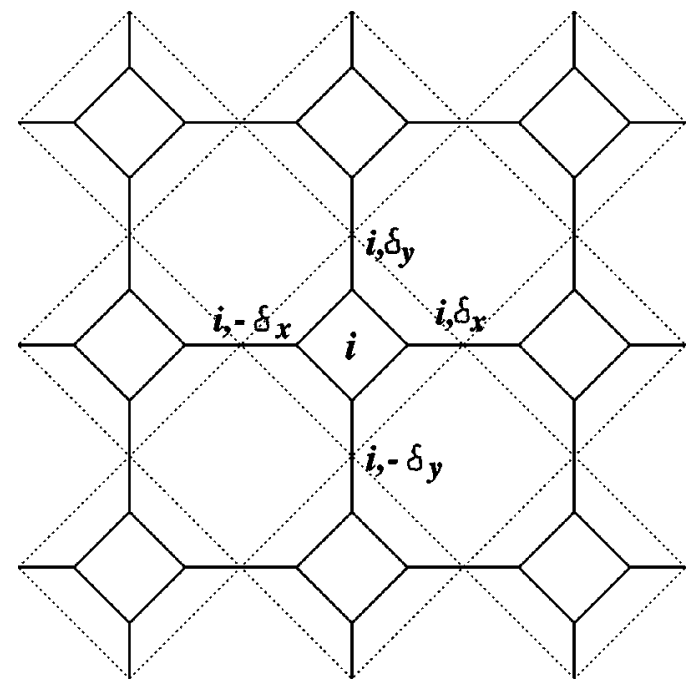

FIG. 1. The $\frac{1}{5}$-depleted lattice. Dotted lines connect nearestneighbor horizontal and vertical bonds.

ingful at long wavelength and a more complete understanding is in high demand. In fact, the only reasonably complete theory is the one by Vojta and Sachdev, ${ }^{13}$ based on the large $N /$ small $S$ saddle point of the $\operatorname{Spl}(2 N) t-J$ model. However, in this large $N$ limit the antiferromagnetism is in the strongly quantum disordered regime, and is therefore at best dual to the renormalized classical Néel order of the stripe phases.

Here we will present an exceedingly simple toy model which seems nevertheless to catch much of the physics discussed in the above. It is similar in spirit to the lattice-boson description of superconductivity and magnetism discussed in Refs. 14 and 15. The pursuit is to construct a model which at the same time describes localized magnetism and local pairing superconductivity. The magnetism is undoubtedly related to strong, Hubbard $U$ type on-site repulsions. This prohibits for obvious reasons on-site paring. The next microscopic length scale available on the lattice is the lattice constant itself: the pairs causing the superconductivity live on the links of the lattice. ${ }^{16}$ If such a link pair is occupied, the sites connected by this link are both occupied by a single electron. In the presence of on-site repulsions these electrons will tend to turn into a spin system. The number fluctuations implied by the superconducting phase order correspond with such an occupied link-pair becoming unoccupied, causing at the same time a dilution of the spin system.

On the square lattice a subtlety keeps a theory with these link pairs as building blocks from being simple. Different from the large $N$ limit with its spin-Peierls order, ${ }^{13}$ the link pairs cause both conceptual problems in describing the state at half filling as well as serious technical problems. As will be discussed in Sec. II, a consistent formulation requires local constraints to be added to the theory in order to exclude tilings of the lattice characterized by multiple occupancies on the sites. This is not necessarily fatal: the theory is bosonic and it might well be that Jastrov projections cure the problem. A central result of this paper is our discovery of a different lattice where these likely nonessential "correlation" problems are absent: the 1/5 depleted lattice shown in Fig. 1. The linkpairs live on the long bonds, while the short bonds only carry spin-spin interactions. As will be further dis- cussed, this model is characterized by an unproblematic classical (in fact, large $d$ ) limit. This allows us to derive in a controlled way a complete semiclassical description.

As discussed in Sec. III, we find a surprisingly rich phase diagram on the classical level containing all phases, which have been up to now suggested in this context, including the large $N$ spin-quantum paramagnets. Perturbing around this classical limit, we address the structure of the semiclassical theory including the universality classes at the various phase transitions (Sec. IV). By fine tuning parameters, we find lines in the phase diagram where the $\mathrm{SO}(5)$ symmetry is approached. However, even at the most symmetric point $\mathrm{SO}(5)$ is not reached: as we will show, the theory becomes $\mathrm{SO}(5)$ symmetric on the classical level but the quantum corrections destroy this symmetry again. As was already pointed out in the context of the $\mathrm{SO}(5)$ symmetric ladders, fine tuning of the on-site repulsions is required to stabilize the full symmetry (Secs. V and VI).

\section{THE MODEL}

\section{A. Correlated superconductivity}

For the strong-coupling description we are aiming at, the microscopic building blocks are electron link pairs, created by the operators

$$
L_{i, \delta}^{\sigma_{1} \sigma_{2 \dagger}}=c_{i, \sigma_{1}}^{\dagger} c_{i+\delta, \sigma_{2}}^{\dagger}
$$

where $\delta$ is a lattice unit-vector, while $i$ labels the sites. Such a link-pair is the typical microscopic object in a strongcoupling theory of $d$-wave superconductivity and the smallest electron pair that can support spin degrees of freedom. Two serious problems arise when trying to construct a model from these operators, one technical and one conceptual. The technical problem is related to the spatial structure of the link pairs, which introduces correlations between pairs centered on different bonds. These correlations show up in the commutation relations of the link operators. Operators along different bonds do not commute if their links share a common site. As a result, the dimension of the link-operator algebra grows with the system size. This makes a simple pseudo-spin description of the charge sector impossible and not much seems to have been gained by going to the strong-coupling limit.

This problem can be avoided by assuming that one can somehow keep track of which electrons belong to a particular pair (this can be ambiguous, for instance, in the case of four electrons sitting in a square). If this is possible, the link-pairs can be described by hardcore boson operators, satisfying $b_{i, \delta}^{\sigma_{1} \sigma_{2}} b_{i, \delta^{\prime}}^{\sigma_{1} \sigma_{2} \dagger}=0$ for $\delta \neq \delta^{\prime}$. Link bosons on different bonds always commute, removing the problem of the infinite-dimensional link algebra. The correlation effects then show up in a different way, however. The hardcore link bosons are spinful generalizations of the quantum dimers. ${ }^{17,18}$ It is well known that even the classical theory of the dimers is a complex combinatorics problem, which was solved for the case of half filling, ${ }^{19}$ but not for general densities. This problem seems unavoidable when one tries to construct a strong-coupling theory for electron pairs with one or the other real space internal structure on the square lattice. 
The conceptual problem is related to the fact that our link pairs carry spin. It concerns the state at half filling. On the square lattice, there are many ways in which the link pairs can be distributed over the lattice to obtain complete covering. Since the half-filling state is a pure spin system, this charge degree of freedom is superfluous. The link-pair model at half filling therefore suffers from a large degeneracy.

In the large-Nt-J model studied by Vojta and Sachdev, ${ }^{13}$ link pairing arises as a result of nearest-neighbor spin-singlet formation, and the pairs are in this case spin-zero dimers. As a result, different link-pair configurations at half filling correspond to different distributions of the singlet spin bonds over the square lattice. These configurations are therefore physically distinct. The spin-Peierls order which is present at half-filling singles out a particular link-pair configuration, breaking the degeneracy.

For a large $S$ type antiferromagnet, however, the spin sector cannot be used to break the degeneracy associated with half filling. Let us therefore consider a model where link pairing arises as a result of charge-charge interactions. In this case, link pairs can have both a singlet and a triplet spin component, allowing for the construction of a half-filling antiferromagnet. Consider a nearest-neighbor attractive interaction $V$, an on-site repulsive interaction $U$ and a longer-range repulsive interaction $U^{\prime}$,

$$
\begin{aligned}
\mathcal{H}= & \sum_{i}\left[-V \sum_{\delta} n_{i} n_{i+\delta}+U n_{i \uparrow} n_{i \downarrow}\right. \\
& \left.+U^{\prime} \sum_{\delta_{1}, \delta_{2} \neq-\delta_{1}} n_{i} n_{i+\delta_{1}+\delta_{2}}\right]+ \text { hopping processes, }
\end{aligned}
$$

where $\delta$ runs over all lattice unit vectors. The attractive interaction $V$ promotes link pairing, while the longer range repulsive interaction $U^{\prime}$ is needed to counteract phase separation in the strong-coupling limit.

At small electron densities, the strong-coupling limit of the above model describes a dilute gas of electron link pairs. Near half filling, it describes a dilute gas of hole link pairs, moving through a spin background. Taking hole pairs and spins, instead of electron pairs, as the elementary building blocks in the strong-coupling limit near half filling, the large degeneracy in the description is avoided. Such a perspective is not entirely satisfactory, however, since the spin sector is in this case represented in a first-quantized form.

The technical problems, related to the spatial correlations between the link pairs, of course remain also for this model. These correlations become important at finite densities away from zero or half filling, severely complicating the strongcoupling analysis of this model. Moreover, the short-range attractive and long-range repulsive interactions will give rise to charge ordering phenomena at intermediate densities, further complicating the physics.

\section{B. Depleted lattice}

The complex spatial correlations between link pairs and the tendency towards charge-ordering at intermediate densities as discussed in the previous subsection can be avoided by formulating the model not on the square lattice, but on the

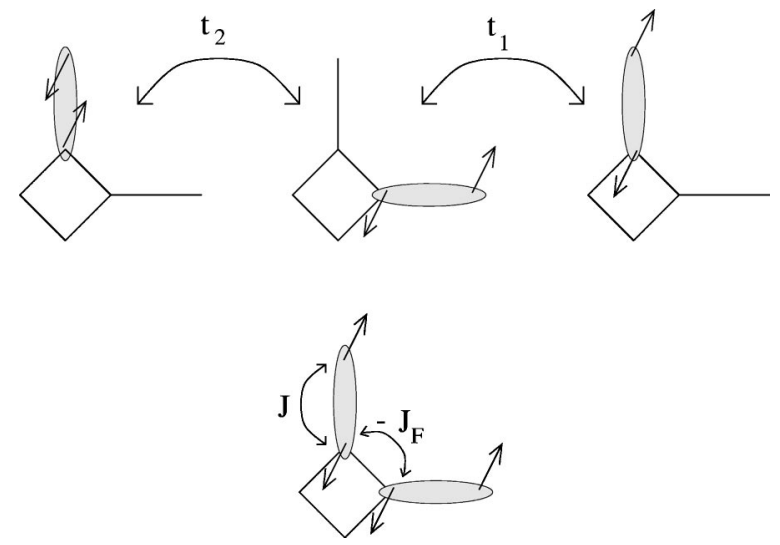

FIG. 2. Hopping processes and spin-spin interactions included in the model.

1/5-depleted lattice, shown in Fig. 1. We arrive at this lattice by expanding the sites of a square lattice to form tilted squares. Along the bonds of the original square lattice, attractive charge-charge interactions are assumed, while onsite repulsive interactions are introduced to promote antiferromagnetism. The electron Hamiltonian of such a model reads

$$
\begin{aligned}
\mathcal{H}= & \sum_{i, \delta}\left[-V n_{1}^{i, \delta} n_{2}^{i, \delta}+U\left(n_{1 \uparrow}^{i, \delta} n_{1 \downarrow}^{i, \delta}+n_{2 \uparrow}^{i, \delta} n_{2 \downarrow}^{i, \delta}\right)\right] \\
& + \text { hopping processes }
\end{aligned}
$$

where the index $i$ labels the square plaquettes, while $(i, \delta)$ denotes the four bonds extending from these plaquettes. The two sites connected by each long bond are numbered 1 and 2 from left to right and from bottom to top. The hopping processes can include hopping along the long and the short bonds, as well as longer-range hopping across the square or the octagonal plaquettes (see Fig. 1). In the large $V$, large $U$ limit, the above model reduces to one describing the physics of spinful link pairs, which reside on the long bonds of the 1/5-depleted lattice. Note that the spatial correlations between these pairs are the same as between point particles on a square lattice. Since the link pairs on different long bonds do not share a common site, the algebra of the link pairs on different bonds decouples and a pseudospin type description of the charge sector becomes possible. Admittedly, this amounts to a rather radical simplification as compared to the square lattice link-pair problem. However, the long wavelength physics we will derive for the depleted lattice might be of a greater generality because of the universality principle. In fact, we suspect that the complexities discussed in the previous subsection will add only tendencies towards charge ordering which can be to some extent discussed separately.

\section{Pair-hopping and spin-spin interactions}

Since the Hamiltonian Eq. (3) should be viewed as a toy model, there is no reason to explicitly derive the strongcoupling description by starting from this Hamiltonian and integrating out the states with unpaired electrons. Instead, we simply formulate another toy model, which describes generic features of the dynamics of bound link pairs on the 1/5- 
depleted lattice. We include the minimal number of processes needed to capture the physics of such a system, making sure that the interactions are consistent with the symmetries of the lattice.

An antiferromagnetic spin-spin interaction $J$ is assumed along the long bonds and a ferromagnetic interaction $J_{F}$ along the short bonds (Fig. 2). This choice allows for an extension of the model to higher dimensions without introducing frustration into the spin system, making it possible to reach the $d \rightarrow \infty$ limit and check the mean-field results there. For $J_{F} \gg J$, the half-filled system becomes equivalent to an $S=2$ antiferromagnet on a square lattice (or $S=d$ on a $d$-dimensional hypercubic lattice). This property will be used to obtain an estimate of the quantum corrections to the saddle-point results obtained in the next section.

A sublattice and an intersublattice hopping process are introduced, with amplitudes $t_{1}$ and $t_{2}$. Both processes move a pair from a horizontal (vertical) bond to a nearest-neighbor vertical (horizontal) bond. The $t_{1}$ process respects the spin ordering, keeping the electrons which form the pair on their original sublattice. The $t_{2}$ process moves the electrons from one sublattice to another, thereby frustrating Néel order.

Including a chemical potential $\mu$, we arrive at the Hamiltonian

$$
\begin{aligned}
\mathcal{H}= & \sum_{i}\left[\sum _ { \sigma _ { 1 } \sigma _ { 2 } } \left\{t_{1}\left(L_{i, \delta_{x}}^{\sigma_{1} \sigma_{2} \dagger}-L_{i,-\delta_{x}}^{\sigma_{2} \sigma_{1}^{\dagger}}\right)\left(L_{i, \delta_{y}}^{\sigma_{1} \sigma_{2}}-L_{i,-\delta_{y}}^{\sigma_{2} \sigma_{1}}\right)\right.\right. \\
& \left.+t_{2}\left(L_{i, \delta_{x}}^{\sigma_{1} \sigma_{2} \dagger}-L_{i,-\delta_{x}}^{\sigma_{2} \sigma_{-}^{\dagger}}\right)\left(L_{i, \delta_{y}}^{\sigma_{2} \sigma_{1}}-L_{i,-\delta_{y}}^{\sigma_{1} \sigma_{2}}\right)+\text { H.c. }\right\} \\
& -J_{F}\left(\vec{s}_{1 i, \delta_{x}}+\vec{s}_{2 i,-\delta_{x}}\right) \cdot\left(\vec{s}_{1 i, \delta_{y}}+\vec{s}_{2 i,-\delta_{y}}\right) \\
& \left.+\sum_{\delta=\delta_{x}, \delta_{y}}\left(\vec{s}_{1 i, \delta} \cdot \vec{s}_{2 i, \delta}-\mu n_{i, \delta}\right)\right],
\end{aligned}
$$

where the same notation has been used as in Eq. (3). A projection operator $P_{i \delta}=\left(1-n_{1 \uparrow}^{i \delta} n_{1 \downarrow}^{i \delta}\right)\left(1-n_{2 \uparrow}^{i \delta} n_{2 \downarrow}^{i \delta}\right)$ has been included in the definition of the link operators $L_{i \delta}^{\sigma_{1} \sigma_{2} \dagger}$, Eq. (1). This enforces the constraint of no double occupancy, which is a result of the large $U$ limit in Eq. (3).

The Hilbert space on one long bond is spanned by five states: unoccupied $(V)$, spin-singlet $(A)$, and spin-triplet $(1,0,-1)$. The operators acting on this space are $5 \times 5$ matrices. Introducing the notation

$$
\left(G_{a b}\right)_{i j}=\delta_{i, a} \delta_{j, b},
$$

the pair creation operators can be written as

$$
\begin{gathered}
L_{\uparrow \uparrow}^{\dagger}=G_{1 V}, \\
L_{\downarrow \downarrow}^{\dagger}=G_{-1 V}, \\
L_{\uparrow \downarrow}^{\dagger}=\frac{1}{\sqrt{2}}\left(G_{0 V}-G_{A V}\right), \\
L_{\downarrow \uparrow}^{\dagger}=\frac{1}{\sqrt{2}}\left(G_{0 V}+G_{A V}\right) .
\end{gathered}
$$

These operators are the equivalent of the pseudo-spins which appear in the strong-coupling negative $U$ Hubbard model. ${ }^{20}$
The operators $G_{\alpha V}, G_{V \alpha}$, and $\frac{1}{2}\left(n_{\alpha}-n_{V}\right)$ form an $S=\frac{1}{2}$ spin algebra $(\alpha=1,0,-1, A)$. Pseudospins with a different spin index $\alpha$ do not commute. In Sec. $\mathrm{V}$, the constraint of no double occupancy is abandoned to allow for the construction of an $\mathrm{SO}(5)$ symmetric version of this model. The operators (6) then become $S=1$ pseudospins and operators with a different index $\alpha$ do commute in this case.

It is convenient to introduce the total spin and the Néel moment of a link pair

$$
\vec{S}_{i, \delta}=\vec{s}_{1 i, \delta}+\vec{s}_{2 i, \delta} ; \quad \overrightarrow{\widetilde{S}}_{i, \delta}=\vec{s}_{1 i, \delta}-\vec{s}_{2 i, \delta},
$$

which are given by

$$
\begin{gathered}
S^{z}=G_{11}-G_{-1-1}, \\
S^{+}=\sqrt{2}\left(G_{10}+G_{0-1}\right), \\
\widetilde{S}^{z}=-G_{A 0}-G_{0 A}, \\
\widetilde{S}^{+}=\sqrt{2}\left(G_{1 A}-G_{A-1}\right),
\end{gathered}
$$

satisfying $\mathrm{SO}(4)$ commutation relations. After absorbing a factor $(-1)^{i_{x}+i_{y}} \operatorname{sgn}(\delta)$ into the triplet states, which induces a staggering of $\overrightarrow{\tilde{S}}$ and $G_{\alpha V}(\alpha=1,0,-1)$, the Hamiltonian takes the form

$$
\begin{aligned}
\mathcal{H}= & \sum_{i} \sum_{\substack{\delta_{1}= \pm \delta_{x} \\
\delta_{2}= \pm \delta_{y}}}\left[\left(t_{1}+t_{2}\right) \sum_{\alpha=1,0,-1}\left(G_{\alpha V}^{i, \delta_{1}} G_{V \alpha}^{i, \delta_{2}}+\text { H.c. }\right)\right. \\
& +\left(t_{1}-t_{2}\right)\left(G_{A V}^{i, \delta_{1}} G_{V A}^{i, \delta_{2}}+\text { H.c. }\right) \\
& \left.-\frac{J_{F}}{4}\left(\vec{S}_{i, \delta_{1}}+\eta_{i} \overrightarrow{\widetilde{S}}_{i, \delta_{1}}\right) \cdot\left(\vec{S}_{i, \delta_{2}}+\eta_{i} \overrightarrow{\widetilde{S}}_{i, \delta_{2}}\right)\right] \\
& +\sum_{i} \sum_{\delta=\delta_{x}, \delta_{y}}\left[\frac{1}{4} J\left(1-n_{V}^{i, \delta}-4 n_{A}^{i, \delta}\right)-\mu\left(1-n_{V}^{i, \delta}\right)\right]
\end{aligned}
$$

where $\eta_{i}=(-1)^{i_{x}+i_{y}}$ is the AF staggering factor. Note that it cannot be absorbed into $\overrightarrow{\widetilde{S}}_{i, \delta}$, since $\left(i, \delta_{x}\right)$ and $\left(i+1,-\delta_{x}\right)$ label the same bond.

\section{MEAN-FIELD ANALYSIS}

A variational Hartree-Fock procedure is used for the mean-field analysis. In the ansatz wave function, the Néel vector is fixed in the $z$ and the total spin in the $x$ direction $(\langle\vec{S}\rangle \cdot\langle\overrightarrow{\widetilde{S}}\rangle=0)$. The pseudospin degrees of freedom of the charge/phase sector are described by an $S=\frac{1}{2}$ spin coherent state

$$
\left|\theta, \psi ; \widetilde{\phi}^{y}, \chi\right\rangle=\sin \theta e^{-i \psi}|V\rangle+\cos \theta\left|\widetilde{\phi}^{y}, \chi\right\rangle,
$$

while the spin degrees of freedom of the pair are contained in $\left|\widetilde{\phi}^{y}, \chi\right\rangle$

$$
\left|\widetilde{\phi}^{y}, \chi\right\rangle=e^{-i \widetilde{\phi}^{y} \widetilde{S}^{y}}(\cos \chi|A\rangle-\sin \chi|0\rangle)
$$

$\left|\widetilde{\phi}^{y}, \chi\right\rangle$ is just the bilayer coherent state $^{21}$ where the global orientation of the two-spin system has been fixed. 

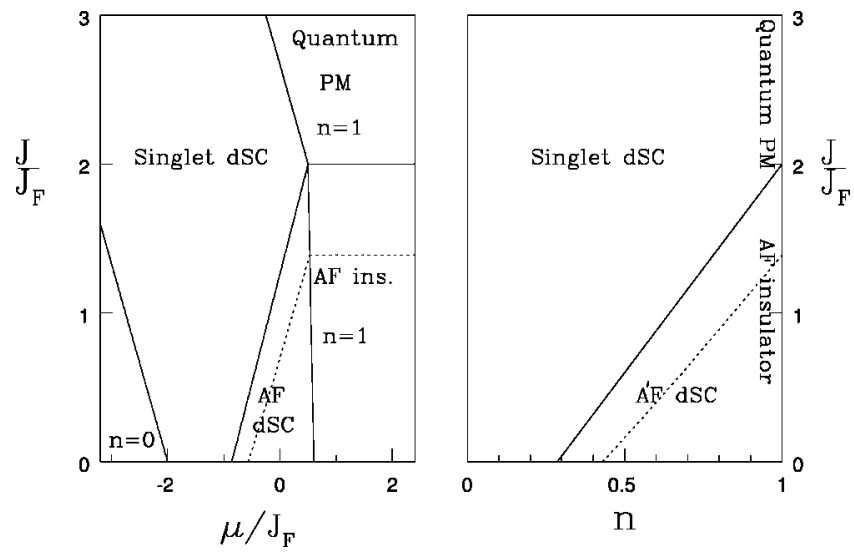

FIG. 3. Mean field phase diagram of $J$ versus $\mu$ and $n$, for $t_{1}$ $>t_{1}^{*} \quad\left(t_{1}=0.4 J_{F}, t_{2}=-0.1 J_{F}\right)$. Bold lines indicate first order transitions. At the dotted line, transversal quantum spin fluctuations destroy the antiferromagnetic order.

We list the expectation-value of a number of quantities with respect to the variational state

$$
\begin{gathered}
n=1-\left\langle n_{V}\right\rangle=\cos ^{2} \theta, \\
\left\langle S^{x}\right\rangle=n \sin 2 \chi \sin \widetilde{\phi}^{y} ; \quad\left\langle S^{y}\right\rangle=\left\langle S^{z}\right\rangle=0, \\
\left\langle\widetilde{S}^{z}\right\rangle=n \sin 2 \chi \cos \widetilde{\phi}^{y} ; \quad\left\langle\widetilde{S}^{x}\right\rangle=\left\langle\widetilde{S}^{y}\right\rangle=0, \\
\left\langle n_{A}\right\rangle=n \cos ^{2} \chi \cos ^{2} \widetilde{\phi}^{y}, \\
\left\langle G_{\alpha V}\right\rangle=\sqrt{n(1-n)} e^{-i \psi}\left\langle\widetilde{\phi}^{y}, \chi \mid \alpha\right\rangle,
\end{gathered}
$$

where $\alpha=1,0,-1, A$ labels the four spin states. The role which the various parameters play can be determined from this list: $\theta$ fixes the pair density; $\widetilde{\phi}^{y}$ determines the relative magnitude of $\langle\vec{S}\rangle$ and $\langle\overrightarrow{\widetilde{S}}\rangle$, while their total magnitude is fixed by $\chi ; \psi$ represents the phase which orders in the superconducting state.

The variational energy is given by

$$
E_{\mathrm{var}}\left(\left\{\theta, \psi ; \widetilde{\phi}^{y}, \chi\right\}_{i, \delta}\right)=\langle\{i, \delta\}|\mathcal{H}|\{i, \delta\}\rangle
$$

where
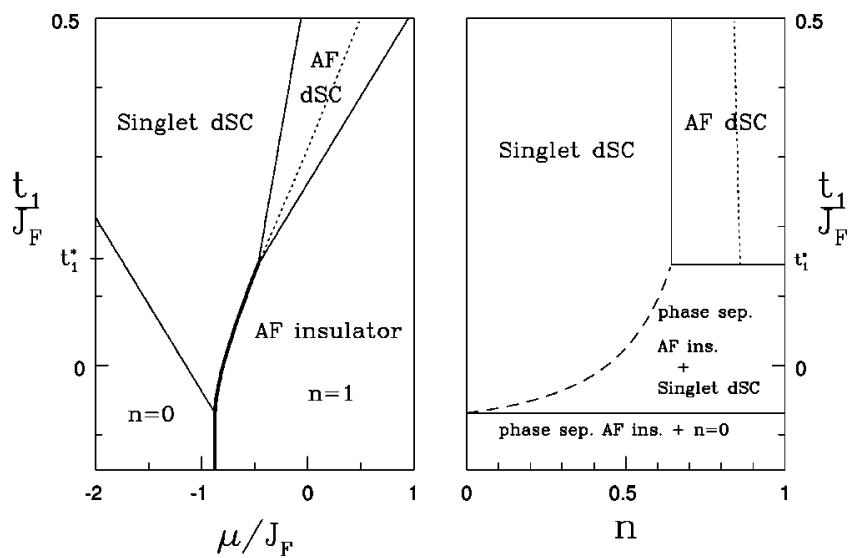

FIG. 4. Mean field phase diagram of $t_{1}$ versus $\mu$ and $n$, for $J$ $<2 J_{F}$ and $t_{2}<J / 8\left(J=J_{F}, t_{2}=-0.1 J_{F}\right)$.
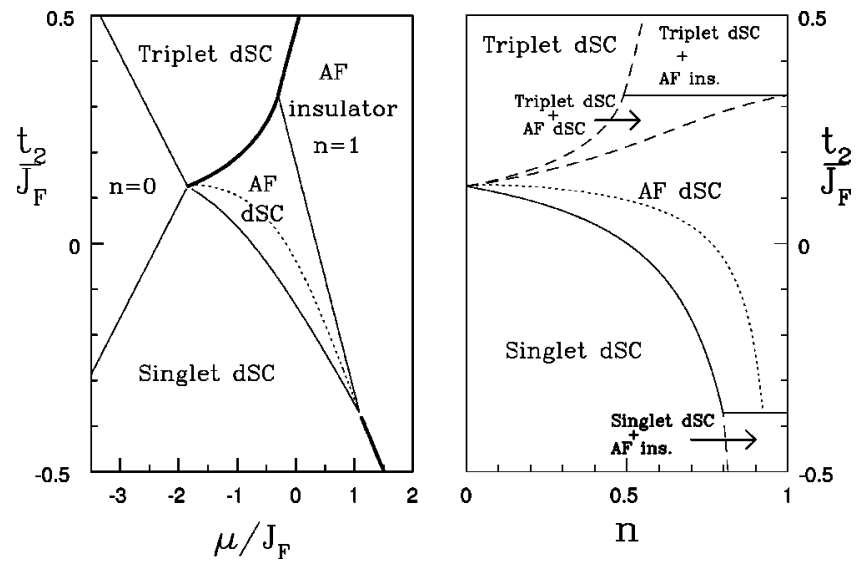

FIG. 5. Mean field phase diagram of $t_{2}$ versus $\mu$ and $n$, for $J$ $<2 J_{F}$ and $t_{1}>\left(4 J_{F}^{2}+J^{2}\right) / 32 J_{F}\left(t_{1}=0.4 J_{F}, J=J_{F}\right)$.

$$
|\{i, \delta\}\rangle=\prod_{i, \delta}\left|\theta, \psi ; \widetilde{\phi}^{y}, \chi\right\rangle_{i, \delta}
$$

In the mean-field analysis, it is assumed that the staggered local magnetization and the charge density are uniform. The phase $\psi_{l}$ is allowed to have a different value on horizontal $\left(\psi^{\mathrm{H}}\right)$ and vertical bonds $\left(\psi^{\mathrm{V}}\right)$. We then arrive at the following mean-field energy:

$$
\begin{aligned}
E_{\mathrm{MF}}= & N\left[\sin ^{2} 2 \theta\left(t_{1}+t_{2}-2 t_{2} \cos ^{2} \chi \cos ^{2} \widetilde{\phi}^{y}\right) \cos \left(\psi^{\mathrm{H}}-\psi^{\mathrm{V}}\right)\right. \\
& -\frac{1}{2} J_{F} \cos ^{4} \theta \sin ^{2} 2 \chi \\
& \left.+\frac{1}{4} J \cos ^{2} \theta\left(1-4 \cos ^{2} \chi \cos ^{2} \widetilde{\phi}^{y}\right)-\mu \cos ^{2} \theta\right]
\end{aligned}
$$

where $N$ denotes the number of long bonds. Minimizing Eq. (15), a variety of mean-field ground states is obtained as a function of the various parameters. The results are summarized in Figs. 3-5 and in Table I. We focus here on the case $t_{1}>0$, for which the superconducting state is typically of $d$-wave type $\left(\psi^{\mathrm{H}}-\psi^{\mathrm{V}}=\pi\right)$. The same phase diagram results for $t_{1} \rightarrow-t_{1}$ and $t_{2} \rightarrow-t_{2}$, but with $s$ wave instead of

TABLE I. Mean-field results for the various phases.

\begin{tabular}{lccc}
\hline \hline Phase & $n$ & $\cos 2 \chi$ & $\cos \widetilde{\phi}^{y}$ \\
\hline Spin-liquid & 1 & 1 & 1 \\
Néel dSC & $\frac{-J-4 \mu-16 t_{1}-8 J t_{2}+64 t_{2}^{2}}{4\left(1-8 t_{1}+16 t_{2}^{2}\right)}$ & $\frac{J+8 t_{2}(n-1)}{2 n}$ & 1 \\
\cline { 2 - 3 } Singlet dSC & $\frac{4 \mu+16\left(t_{1}-t_{2}\right)+3 J}{32\left(t_{1}-t_{2}\right)}$ & 1 & 1 \\
Triplet dSC & $\frac{J-4 \mu-16\left(t_{1}+t_{2}\right)}{4-32\left(t_{1}+t_{2}\right)}$ & 0 & 0 \\
AF & 1 & $\frac{J}{2}$ & 1
\end{tabular}


$d$-wave phase order. For simplicity, $J, t_{1}, t_{2}$, and $\mu$ are expressed in units of $J_{F}$ from here on.

At half filling, the physics is determined by the competition between the antiferromagnetic and the ferromagnetic spin-spin interaction. While the first promotes singlet formation along the horizontal and vertical bonds, the second favors large local magnetic moments. $J$ therefore tunes the singlet density in the ground state at half filling. For $J \ll 1$, the system has full Néel order with $\left\langle n_{A}\right\rangle=\frac{1}{2},|\langle\overrightarrow{\widetilde{S}}\rangle|=1$. The singlet density increases linearly with $J$ up to $\left\langle n_{A}\right\rangle=1$ at $J$ $=2$, where the staggered magnetization vanishes in a second order transition to a quantum paramagnet phase (Fig. 3).

For densities smaller than one, the two hopping processes begin to play a role. Since the case of a uniform charge distribution is considered and since all electrons are paired in the strong-coupling limit, all variational states with a noninteger electron density exhibit superconductivity. The superconducting order parameter depends on the electron density as $|\Delta| \sim \sqrt{n(1-n)}$, see Eq. (12).

The value of the hopping amplitude $t_{1}$ determines the nature of the transition from the antiferromagnetic insulator at half filling to the singlet superconductor at lower densities. For small $t_{1}$, this transition is first order as a function of $\mu$, giving rise to a region of antiferromagnet/superconductor phase separation in the $t_{1}-n$ phase diagram (Fig. 4). At $t_{1}$ $=t_{1}^{*}=\frac{1}{8}+2 t_{2}^{2}$, the first order line splits into two second order lines. A region opens up in which the system has both antiferromagnetic spin order and superconductivity. In this antiferromagnetic superconductor (AFSC) phase, the electrons which carry the superconducting order parameter are at the same time responsible for the antiferromagnetism. This state is most easily visualized by thinking of a small density of nearest-neighbor hole pairs being doped into a half-filling antiferromagnet. If these hole pairs are most mobile along the diagonals of the square lattice, where their movement does not disturb the AF spin order, they can delocalize and in that way give rise to superconductivity without at the same time destroying the antiferromagnetic order. The condition that diagonal pair hopping has to dominate to get an AFSC phase on the square lattice is reflected by the condition $t_{1}$ $>t_{1}^{*}$ for the present model.

There are three ways in which the spin order parameter in the AFSC phase is suppressed through the doping with hole pairs. The simplest one corresponds with the dilution of the antiferromagnet by the removal of spins. More interestingly, the interpair spin-spin interaction $J_{F}$ scales with the pairdensity squared, while the intrapair spin-spin interaction $J$ scales linearly with $n$. As a result, the ferromagnetic interaction is suppressed by a factor $n$ relative to $J$, pushing the ratio $J / J_{F}$ closer to its critical value and reducing the magnetic moment per pair. Finally, the hopping process $t_{2}$ frustrates the Néel order, provided that $\operatorname{sgn}\left(t_{2}\right)=-\operatorname{sgn}\left(t_{1}\right)$ (the other case is discussed below). The increase of the singlet density per pair due to the last two processes results in a transition to the singlet superconductor at $n=n_{c}=\left(J-8 t_{2}\right) /\left(2-8 t_{2}\right)$.

Since the $t_{2}$ process amounts to a $t_{1}$-type hop with an additional interchange of the two electrons forming the pair, it picks up a minus sign when acting on a pair in the antisymmetric spin-singlet state. Suppose that $t_{1}$ and $t_{2}$ have the same sign. A singlet pair, through the $t_{2}$ process, then frus- trates the phase ordering as favored by the $t_{1}$ hop. To reduce this frustration, the singlet content of the pairs is suppressed as $t_{2}$ is increased, enhancing the spin ordering in the AFSC phase. Eventually, a first order transition occurs to a ferromagnetically ordered triplet superconductor phase, where the singlet density is reduced to zero (Fig. 5).

If $t_{1}$ and $t_{2}$ have opposite sign, the triplet component is suppressed through the same process and the $t_{2}$ hop reduces the spin-order in the AFSC phase. Note that $t_{2}$ causes a positive shift of the critical value $t_{1}^{*}$ regardless of its sign. This reflects the fact that on-sublattice hopping must dominate in order for an AFSC phase to occur $\left(t_{1}>t_{1}^{*}\right.$ implies $t_{1} \geqslant\left|t_{2}\right|$, where the equal sign occurs for $\left|t_{2}\right|=\frac{1}{4}$ ).

It should be verified that the saddle-point solution becomes exact in the limit $d \rightarrow \infty$. To reach this limit, the model has to be formulated in arbitrary dimension. The $d$-wave phase order then posses a problem, since it cannot be generalized to dimensions higher than 2. However, the Hamiltonian of the two-dimensional (2D) system is invariant under a simultaneous sign change of $t_{1}, t_{2}$, and $G_{\alpha V}^{i, \pm} \delta_{x}$, which implies that $d$ - and $s$-wave order are equivalent for the 2D model. We therefore flip the sign of $t_{1}$ and $t_{2}$ and study the $d \rightarrow \infty$ limit for the s-wave ordered state. In order to keep the energy finite, $J_{F}, t_{1}$, and $t_{2}$ are scaled with $1 / d$ while taking the limit. The variation of the energy is found to be of order $1 / d$ for the saddle-point solution. Since it vanishes at large $d$, the mean-field groundstate indeed becomes an eigenstate of the system in this limit.

\section{TRANSVERSAL SPIN FLUCTUATIONS}

In the AFSC phase, both the U(1) phase and the SU(2) spin symmetry are spontaneously broken. As a result, the system has two spin-wave modes and one phase Goldstone mode. These gapless modes dominate its long-wavelength physics. Since they decouple, the phase and spin degrees of freedom of the system may be treated separately at sufficiently large length scales.

The physics of the phase sector is equivalent to that of an $X Y$-spin model in an external magnetic field, which has a dynamical critical exponent $z=2$. The $T=0$ system is therefore effectively at its upper critical dimension $d=2+z=4$. Because of the high effective dimensionality, phase fluctuations only give small correction to the zero-temperature mean-field results for the insulator-superconductor transition. ${ }^{22}$

The long-wavelength behavior of the spin sector in the AFSC phase is characterized by a critical exponent $z=1$. Hence, at $T=0$, the spin sector lives effectively in three dimensions and fluctuation effects can be significant. The long-wavelength spin physics is described by an effective nonlinear sigma model. ${ }^{23,24}$ This model contains one coupling constant $g_{0}$ which is a measure of the quantum fluctuations in the system. At a critical value of $g_{0}$, the spin system undergoes a quantum phase transition from a Néel ordered state to a quantum paramagnet. For the present model, $g_{0}$ is expected to diverge at the mean-field transitions to the singlet superconductor and the paramagnetic insulator. ${ }^{21}$ Transversal fluctuations are therefore expected to 
significantly reduce the region in the phase diagram where $\mathrm{AF}$ order is stable.

The coupling constant of the effective non-linear sigma model depends on the bare values of the spin stiffness and the perpendicular susceptibility, which are properties of the microscopic model. The mean-field expressions for these quantities can serve as an estimate for their bare value. ${ }^{21}$ These expressions are derived below for the present model.

We define the perpendicular susceptibility as the induced magnetization per square plaquette (containing four spins) by a vanishing magnetic field applied perpendicular to the direction of antiferromagnetic ordering. It is calculated by adding a magnetic field term to the mean-field energy Eq. (15),

$$
H \sum_{i, \delta=\delta_{x}, \delta_{y}}\left\langle S_{i, \delta}^{x}\right\rangle=N H \cos ^{2} \theta \sin 2 \chi \sin \widetilde{\phi}^{y}
$$

and subsequently minimizing the energy. This yields

$$
\begin{aligned}
\chi_{\perp}=\lim _{H \rightarrow 0} \frac{2\left\langle S^{x}\right\rangle}{H}=\frac{2(1-\cos 2 \chi) n}{J-8 t_{2}(1-n)} \\
\quad \Rightarrow\left\{\begin{array}{l}
\chi_{\perp}^{\mathrm{AF}}=\frac{2-J}{J}, \\
\chi_{\perp}^{\mathrm{AFSC}}=\frac{n\left(2-8 t_{2}\right)-J+8 t_{2}}{J-8 t_{2}(1-n)} .
\end{array}\right.
\end{aligned}
$$

The susceptibility vanishes at the transitions to the quantum paramagnet and the singlet superconductor phase. It has a divergence at $n=1-J / 8 t_{2}$, which is interrupted by the first order transition to the triplet superconductor phase (Fig. 5) (the line where $\chi_{\perp}$ diverges and the first-order line approach each other for small $n$ ).

For $n=1$ and $J_{F} \gg J$, the four spins around each square plaquette lock into a symmetric state. The spin operators can then be replaced by $\frac{1}{4}$ times the total spin operator on the plaquette. The resulting Hamiltonian describes an $S=2$ antiferromagnet on a square lattice, with a spin-spin coupling $J_{\text {eff }}=\frac{1}{16} J$. Such a system has a mean-field perpendicular susceptibility $\chi_{\perp}=1 / 8 J_{\text {eff }}=2 / J^{23}$ (where the lattice spacing, in this case the distance between neighboring square plaquettes, is set to one). The above result for the susceptibility indeed reduces to this expression for $n=1$ and $J \ll 1$.

To determine the spin stiffness, the configuration shown in Fig. 6 is considered. It has a slow twist in the spin order parameter along the $x+y$ direction. The stiffness gives the lowest-order correction to the ground state energy due to this twist. $^{25}$

At each antiferromagnetic bond along the direction of the twist, the spins have been rotated over an angle $\alpha \delta \phi$ in the $X Z$ plane, at each ferromagnetic bond over an angle (1 $-\alpha) \delta \phi$. This configuration is described by the variational state Eq. (10), where the spin part is given by

$$
e^{i l \delta \phi S^{y}}\left|\widetilde{\phi}^{y}=\frac{1}{2} \alpha \delta \phi, \quad \chi=\chi_{\mathrm{MF}}\right\rangle,
$$

with the index $l$ labeling the bonds along the twist.

The antiferromagnetic interaction energy of this state is given by [compare with last line in Eq. (15)]

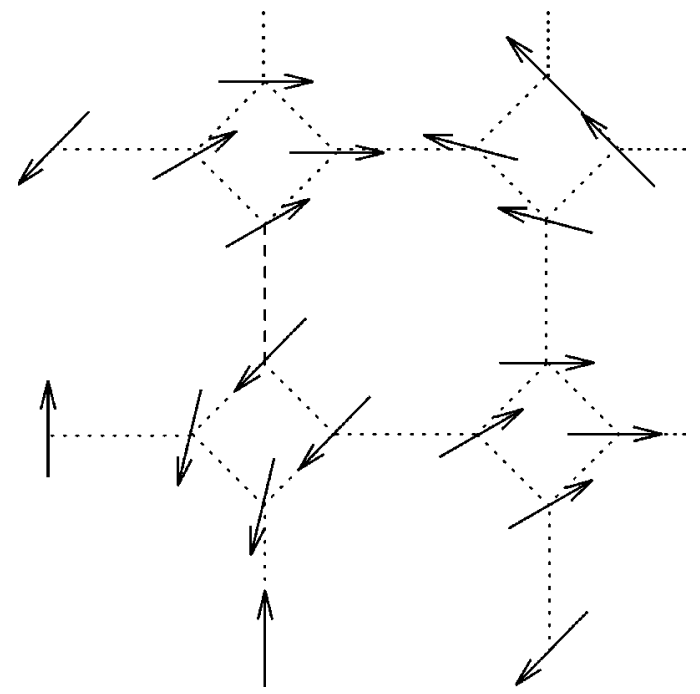

FIG. 6. A spin configuration with a twist along the $x+y$ direction.

$$
\begin{aligned}
& \frac{1}{4} J N \cos ^{2} \theta\left[1-4 \cos ^{2} \chi \cos ^{2}\left(\frac{1}{2} \alpha \delta \phi\right)\right] \\
& \simeq E_{A F}(\delta \phi=0)+\frac{1}{4} J N \alpha^{2} \delta \phi^{2} \cos ^{2} \theta \cos ^{2} \chi
\end{aligned}
$$

while the ferromagnetic energy is simply reduced by a factor $\cos (1-\alpha) \delta \phi$ per twisted ferromagnetic bond. The phaseordering energy also contributes to the spin stiffness. Along the twist, we have ( $d$-wave order)

$$
\begin{aligned}
& \sum_{\alpha=1,0,-1}\left\langle G_{\alpha V}^{l}\right\rangle\left\langle G_{\alpha V}^{l+1}\right\rangle \\
& =-\sum_{\alpha=1,0,-1} \frac{1}{4} \sin ^{2} 2 \theta\left\langle\overrightarrow{\mathbf{\Omega}}\left|e^{-i \boldsymbol{l} \boldsymbol{\delta} \phi S^{y}}\right| \alpha\right\rangle \\
& \times\left\langle\alpha\left|e^{i(l+1) \delta \phi S^{y}}\right| \overrightarrow{\mathbf{\Omega}}\right\rangle \\
& =-\frac{1}{4} \sin ^{2} 2 \theta\left\langle\vec{\Omega}\left|(1-|A\rangle\langle A|) e^{i \delta \phi S^{y}}\right| \vec{\Omega}\right\rangle \\
& \simeq-\left\langle G_{A V}^{l}\right\rangle\left\langle G_{V A}^{l+1}\right\rangle-\frac{1}{4} \sin ^{2} 2 \theta \\
& \times(1-\frac{1}{2} \delta \phi^{2} \underbrace{\left\langle\vec{\Omega}\left|S^{y}\right| \vec{\Omega}\right\rangle}_{\sin ^{2} \chi}), \\
& \begin{aligned}
\left\langle G_{A V}^{l}\right\rangle\left\langle G_{V A}^{l+1}\right\rangle & =-\frac{1}{4} \sin ^{2} 2 \theta \cos ^{2} \chi \cos ^{2}\left(\frac{1}{2} \alpha^{2} \delta \phi^{2}\right) \\
& \simeq-\frac{1}{4} \sin ^{2} 2 \theta \cos ^{2} \chi\left(1-\frac{1}{4} \alpha^{2} \delta \phi^{2}\right) .
\end{aligned}
\end{aligned}
$$

Taking these contributions together, the energy-increase due to the twist in the spin order-parameter is found to be 


$$
\begin{aligned}
\Delta E= & \frac{1}{8} N \delta \phi^{2}\left(2 \sin ^{2} 2 \theta\left[\left(t_{1}+t_{2}\right) \sin ^{2} \chi-t_{2} \alpha^{2} \cos ^{2} \chi\right]\right. \\
& \left.+\sin ^{2} 2 \chi \cos ^{4} \theta(1-\alpha)^{2}+2 J \alpha^{2} \cos ^{2} \theta \cos ^{2} \chi\right) .
\end{aligned}
$$

The distribution of the total twist over the two types of bonds is obtained by minimizing this energy with respect to $\alpha$, which yields

$$
\alpha_{0}=\frac{n(1-\cos 2 \chi)}{n(1-\cos 2 \chi)+J-4 t_{2}(1-n)} .
$$

For $J_{F}$ much larger than $J$ and $t_{2}$ (or just $J$ for $n=1$ ), the twist is entirely localized on the antiferromagnetic bonds, as expected. At the transition to the spin disordered phases, it is localized on the ferromagnetic bonds.

The stiffness now follows from

$$
\Delta E\left(\alpha_{0}\right)=\frac{N}{2} \delta \phi^{2} \rho_{s}
$$

For the half-filling antiferromagnet, we obtain

$$
\rho_{s}^{\mathrm{AF}}=\frac{1}{8} J(2-J)
$$

while for the AFSC phase the stiffness is given by

$$
\begin{aligned}
\rho_{s}^{\mathrm{AFSC}}= & \frac{n\left(2-8 t_{2}\right)-J+8 t_{2}}{8 J+16 n}\left\{2 J n+J^{2}+4(1-n)\right. \\
& \left.\times\left[J\left(t_{1}-2 t_{2}\right)+2 t_{1} n+8 t_{2}^{2}(1-n)\right]\right\} .
\end{aligned}
$$

As with the susceptibility, the stiffness vanishes at the transition to a spin-disordered phase. It reduces to the $S=2$, $J_{\text {eff }}=\frac{1}{16} J$ form for $J \gg J_{F}$ at half filling.

The bare coupling constant of the nonlinear sigma model is given by $g_{0}=\left(\rho_{s} \chi_{\perp}\right)^{-1 / 2}$. As expected, it diverges at the transitions to the singlet superconductor and quantum paramagnet, since both the susceptibility and the stiffness vanish in these phases. In order to obtain a more precise estimate of $g_{0}$, its value is shifted by a constant factor such that it agrees with the result for the $S=2$ antiferromagnet at $n=1, J_{F}$ $\gg J$. The bare coupling for the square lattice $S=2$ antiferromagnet can be determined from spin-wave results for the renormalized spin-wave velocity and perpendicular susceptibility, using the one-loop expression ${ }^{24}$

$$
\frac{g_{0}}{4 \pi}=\frac{1}{1+4 \pi \chi_{\perp} c / \hbar \Lambda}
$$

where $\Lambda=2 \sqrt{\pi} / a$, with $a$ the lattice spacing. Using the spinwave results of Igarashi, ${ }^{25}$ we obtain

$$
g_{0}^{S=2} \simeq 3.85 .
$$

For the half-filling antiferromagnet, the bare coupling constant is given by

$$
g_{0}=g_{0}^{S=2} \frac{2}{2-J},
$$

while we find for the AFSC phase

$$
g_{0}=g_{0}^{S=2} \frac{2 \sqrt{(2 n+J)\left[J-8 t_{2}(1-n)\right]}}{n\left(2-8 t_{2}\right)-J+8 t_{2}} \frac{1}{\sqrt{2 n J+J^{2}+4(1-n)\left[J\left(t_{1}-2 t_{2}\right)+2 t_{1} n+8 t_{2}^{2}(1-n)\right]}} .
$$

The order-disorder transition at $g_{0}=g_{c}=4 \pi$ is indicated by a dotted line in the mean-field phase diagrams, Figs. 3-5. It is found that transversal spin fluctuations significantly reduce the parameter range over which the Néel-ordered phases are stable, without changing the topology of the zerotemperature phase diagram.

At nonzero but low temperatures, the quantum nonlinear sigma model predicts $z=1$ quantum critical behavior in a parameter region around the AFSC to SC transition line. ${ }^{24}$ The width of this region grows as $\left|g_{0}-4 \pi\right| \sim T^{-\nu}$, with $\nu$ $=0.7$ the correlation-length critical exponent of the $3 \mathrm{D}$ Heisenberg model. This type of finite temperature behavior, where temperature becomes the only energy scale in the system, has been reported for the underdoped cuprates by a number of authors. ${ }^{26-28}$

Finally, we note that in the present model the superconductivity onset temperature is completely determined by phase fluctuations. This is a trivial consequence of the fact that we focussed on the strong-pairing limit. Nevertheless, it is consistent with recent analyses of the dependence of $T_{c}$ on the zero-temperature phase stiffness and on the number of closely spaced layers in the superconductor material, which point to a dominant role of finite-temperature phase fluctuations in determining $T_{c}{ }^{29}$

\section{SO(5) SYMMETRIC POINT}

The AFSC phase has an interesting property. Let us consider the $\mathrm{SO}(5)$ superspin-vector ${ }^{11}$ for this model

$$
\vec{N}_{\mathrm{P}}=\left(\frac{1}{2}\left(G_{A V}+G_{V A}\right), \frac{1}{2} \overrightarrow{\widetilde{S}}, \frac{1}{2 i}\left(G_{A V}-G_{V A}\right)\right) \text {. }
$$

The label $P$ indicates that $\vec{N}_{\mathrm{P}}$ is defined in the projected Hilbert space, where double site-occupancy is forbidden. The mean-field expectation value of $\vec{N}_{\mathrm{P}}$ satisfies

$$
\left.\frac{\partial\left\langle\vec{N}_{\mathrm{P}}\right\rangle^{2}}{\partial n}\right|_{t_{2}=-1 / 4}=0 .
$$


Hence, at the mean-field level and for this particular choice of $t_{2}$, the AFSC phase can be characterized by an $\mathrm{SO}(5)$ order parameter which has components both in the superconducting and in the antiferromagnetic subspace, and which is rotated from the AF to the SC direction as the hole density is increased. As one approaches the tricritical point, the AFSC states with different $n$ become degenerate (Fig. 3) and the mean-field state becomes invariant under rotations of $\vec{N}_{\mathrm{P}}$. For $t_{2}=-\frac{1}{4}$ the tricritical-critical point is located at $t_{1}=t^{*}$ $=\frac{1}{4}, \mu=\mu^{*}=J / 4$.

It should perhaps come as no surprise that we find a "mean-field SO(5) symmetry" for this model. The special lattice used here has two orbitals per unit cell, which seems to be one of the requirements for constructing an SO(5) symmetric model with short-range interactions. ${ }^{30}$ This can be understood from the fact that the minimum number of sites required for the electron Hilbert-space in which an $\mathrm{SO}(5)$ representation can be constructed is 2 (since the $\pi$ operators are spin-1, charge 2 objects). Two-leg spin ladders have a natural two-site unit, the rung, on which the $\mathrm{SO}(5)$ order parameter can be defined. The lattice used here also has such a unit: the long bond. To formulate a short-range $\mathrm{SO}(5)$ model on the square lattice, one either has to break the lattice symmetry, or to involve a certain amount of coarse graining, which means that the resulting $\mathrm{SO}(5)$ description is effective rather than microscopic.

In the following, an exact $\mathrm{SO}(5)$ symmetric point is derived for the present model. The procedure used is similar to that for the $\mathrm{SO}(5)$ symmetric ladder. ${ }^{31}$ At the mean-field $\mathrm{SO}(5)$-point, the Hamiltonian is given by

$$
\mathcal{H}=\mathcal{H}_{0}+\mathcal{H}_{1}
$$

where

$$
\begin{gathered}
\mathcal{H}_{0}=-\sum_{i} \sum_{\substack{\delta_{1}= \pm \delta_{x} \\
\delta_{2}= \pm \delta_{y}}} \vec{N}_{\mathrm{P}}^{i, \delta_{1}} \cdot \vec{N}_{\mathrm{P}}^{i, \delta_{2}}-J \sum_{i} \sum_{\delta=\delta_{x}, \delta_{y}} n_{A}^{i, \delta}, \\
\mathcal{H}_{1}=\sum_{i} \sum_{\substack{\delta_{1}= \pm \delta_{x} \\
\delta_{2}= \pm \delta_{y}}} \frac{1}{4}\left[\overrightarrow{\widetilde{S}}_{i, \delta_{1}} \cdot \overrightarrow{\widetilde{S}}_{i, \delta_{2}}+\eta_{i}\right. \\
\left.\quad \times\left(\vec{S}_{i, \delta_{1}} \cdot \overrightarrow{\widetilde{S}}_{i, \delta_{2}}+\overrightarrow{\widetilde{S}}_{i, \delta_{1}} \cdot \vec{S}_{i, \delta_{2}}\right)\right],
\end{gathered}
$$

absorbing a $d$-wave staggering into the $\left|V_{i, \delta}\right\rangle$ state.

The second term, $\mathcal{H}_{1}$, is manifestly not invariant under rotations of $\vec{N}_{P}$. After decoupling the operators on different bonds with respect to the order-parameters for superconductivity and antiferromagnetism, this term vanishes and therefore the symmetry breaking does not show up at the meanfield level. As Eder et al. pointed out, ${ }^{14}$ the first and the fourth component of $\vec{N}_{\mathrm{P}}$ are rotated into each other by transforming the zero-magnetization triplet state $|0\rangle$ into the holepair state $|V\rangle$. This transformation leaves the singlet density $n_{A}$ invariant. Since one may assume in mean-field that all components of $\left\langle\vec{N}_{\mathrm{P}}\right\rangle$ vanish except the first and the fourth (spontaneous symmetry breaking selects a preferred direction in the spin and phase sector) the decoupled mean-field Hamiltonian is invariant under this transformation. This im- plies that the $d \rightarrow \infty \mathrm{SO}(5)$ symmetry is not only present in the zero-temperature ground state, but also at finite temperatures, where higher energy levels are thermally occupied.

As a first step towards an $\mathrm{SO}(5)$-symmetric Hamiltonian $\mathcal{H}_{1}$ is subtracted from $\mathcal{H}$. This introduces second- and thirdneighbor spin-spin interactions into the model.

The second term in $\mathcal{H}_{0}$ is $\mathrm{SO}(5)$ invariant (this is discussed below). The first term is invariant under rotations of $\vec{N}_{P}$, but this does not imply that it is $\mathrm{SO}(5)$ symmetric. There is no representation of the $\mathrm{SO}(5)$ algebra on the projected Hilbert space under which $\vec{N}_{\mathrm{P}}$ transforms as a vector. The rotation symmetry is therefore broken at the quantum level. In a recent article, ${ }^{15}$ Zhang et al. show that mean-field $\mathrm{SO}(5)$ symmetry always remains when a projection to the lower Hubbard band is applied to a system with full $\mathrm{SO}(5)$ symmetry. Here we work backwards: mean-field SO(5) symmetry being established, we deduce a model with full $\mathrm{SO}(5)$ symmetry by lifting the constraint of no double site occupancy.

The basis of the single-bond Hilbert space is extended to include the doubly occupied state $|D\rangle$. It now consists of one $\mathrm{SO}(5)$ singlet $(A)$ and one $\mathrm{SO}(5)$ quintet (spin-triplet, $D$ and $V)$. The details of this representation of the $\mathrm{SO}(5)$ algebra are briefly discussed in Appendix A. We introduce an on-site repulsion $U \sum_{i \delta} n_{D i \delta}$. The general Hamiltonian on the unprojected Hilbert space is given by

$$
\begin{aligned}
\mathcal{H}= & \sum_{i} \sum_{\begin{array}{c}
\delta_{1}= \pm \delta_{x} \\
\delta_{2}= \pm \delta_{y}
\end{array}}\left[4\left(t_{1}+t_{2}\right) \sum_{\alpha} \vec{\pi}_{i, \delta_{1}}^{\alpha} \cdot \vec{\pi}_{i, \delta_{2}}^{\alpha}\right. \\
& +\left(t_{1}-t_{2}\right) \vec{\Delta}_{i, \delta_{1}} \cdot \vec{\Delta}_{i, \delta_{2}} \\
& \left.+\frac{1}{4}\left(\vec{S}_{i, \delta_{1}}+\eta_{i} \overrightarrow{\widetilde{S}}_{i, \delta_{1}}\right) \cdot\left(\vec{S}_{i, \delta_{2}}+\eta_{i} \overrightarrow{\widetilde{S}}_{i, \delta_{2}}\right)\right] \\
& -\sum_{i, \delta=\delta_{x}, \delta_{y}}\left[J n_{A}^{i, \delta}+\left(\frac{J}{4}-U+\mu\right) n_{D}^{i, \delta}-\left(\mu-\frac{J}{4}\right) n_{V}^{i, \delta}\right],
\end{aligned}
$$

where $\vec{\Delta}=(\operatorname{Re} \Delta, \operatorname{Im} \Delta), \vec{\pi}^{\alpha}=\left(\operatorname{Re} \pi^{\alpha}, \operatorname{Im} \pi^{\alpha}\right)$ (see Appendix A). The value of $U$ has to be fine-tuned in order to obtain $\mathrm{SO}(5)$ symmetry on a single bond. The resulting constraint is $\mu=J / 4$ as before, but in addition $U=J / 2$. Note that this is more restrictive than the local constraint for the ladder model, ${ }^{31}$ which leaves two free parameters. Since we only consider states of paired electrons, this model has fewer local $\mathrm{SO}(5)$ invariants than the ladder.

To establish $\mathrm{SO}(5)$ symmetry of the inter-pair interactions, one now has to take $t_{1}=-t_{2}=\frac{1}{8}$. After subtraction of $\mathcal{H}_{1}$, this yields the Hamiltonian

$$
\mathcal{H}_{\mathrm{SO}(5)}=-\frac{1}{4} \sum_{\langle l, m\rangle} \vec{N}_{l} \cdot \vec{N}_{m}-J \sum_{l} n_{A}^{l} \text {, }
$$

where $l$ and $m$ run over the square lattice spanned by the long bonds (dotted lines in Fig. 1). The unprojected $\mathrm{SO}(5)$ superspin $\vec{N}$ is given by Eq. (A1). The local $\mathrm{SO}(5)$ invariant $n_{A}$ is related to the length of the superspin through $\vec{N}^{2}=1+4 n_{A}$. 
Note that Eq. (36) is not the most general $\mathrm{SO}(5)$ symmetric Hamiltonian which could be formulated. In principle, there can be an additional term of the form

$$
\begin{aligned}
& \lambda \sum_{\langle l, m\rangle} \sum_{a<b} L_{a b}^{l} L_{a b}^{m} \\
& \quad=\lambda \sum_{\langle l, m\rangle}\left[\sum_{\alpha} \vec{\pi}_{l}^{\alpha} \cdot \vec{\pi}_{m}^{\alpha}+\vec{S}_{i, \delta_{1}} \cdot \vec{S}_{i, \delta_{2}}+Q_{i, \delta_{1}} Q_{i, \delta_{2}}\right]
\end{aligned}
$$

which is also an $\mathrm{SO}(5)$ invariant. The charge-charge interaction $Q_{i, \delta_{1}} Q_{i, \delta_{2}}$ was omitted from the present analysis and a term of this form therefore does not appear at the symmetric point.

The projected $\mathrm{SO}(5)[p \mathrm{SO}(5)]$ symmetry at $U \rightarrow \infty$ evolves from the true $\mathrm{SO}(5)$ symmetric point at fine-tuned $U$ in the following way. Let us assume we have $t_{1}=-t_{2}=t$ and $U=J / 2+\bar{U}$

$$
\mathcal{H}=-\sum_{\langle l, m\rangle}\left(2 t \vec{\Delta}_{l} \cdot \vec{\Delta}_{m}+\frac{1}{4} \overrightarrow{\widetilde{S}}_{l} \cdot \overrightarrow{\widetilde{S}}_{m}\right)+\sum_{l}\left(\bar{U} n_{D}^{l}-J n_{A}^{l}\right) .
$$

The superspin has no preferred global direction for $\bar{U}=0, t$ $=\frac{1}{8}$. Since the AF ground state does not have a $|D\rangle$ component, while the SC does, a small positive $\bar{U}$ will flop the superspin to the AF direction. The energy-difference between the AF and the SC state can be compensated by an increase in $t$. For $\bar{U} \rightarrow \infty$, this procedure shifts the superspinflop point from $t=\frac{1}{8}$ to $t=\frac{1}{4}$, with the SC ground state now having $\left\langle n_{D}\right\rangle=0$. The shift in $t$ is accounted for by the different relative normalization of $\vec{\Delta}$ and $\overrightarrow{\widetilde{S}}$ in the definitions of $\vec{N}_{P}$ and $\vec{N}$.

\section{COLLECTIVE MODES}

In the above, it was shown that the intersublattice hopping $t_{2}$, which couples the spin and charge dynamics in our model, plays a crucial role in establishing the $\mathrm{SO}(5)$ symmetry. This symmetry only emerges at the mean-field level for a specific value of $t_{2}$. To further investigate the role of this hopping process, the collective modes in the antiferromagnetic and spin-disordered phases are analyzed. For $t_{2}=0$, it is found that a decoupled spin/charge perspective suffices to understand these modes, as one would expect. In this case, the superconductivity does not affect the collective spin modes of the system.

This changes for nonzero $t_{2}$. Although the dispersion relations do not change qualitatively, the interpretation of the modes does. Most strikingly, the gapped spin-magnon mode of the singlet SC phase acquires a $\pi$-mode component. This mode softens at the transition to the AFSC phase and becomes a pure, acoustic $\pi$-mode as the system is tuned towards $\mathrm{pSO}(5)$ symmetry.

The mode spectrum for systems with $\mathrm{pSO}(5)$ symmetry was analyzed by Zhang et al. ${ }^{15}$ We reproduce their results for the present model and investigate the influence of further $\mathrm{SO}(5)$ symmetry-breaking terms.

\section{A. Random phase approximation}

The collective modes of the system are studied in the random phase approximation (RPA). ${ }^{34}$ They are obtained from the equations of motion of the operators $G_{\alpha \beta}$, which are given by

$$
i \partial_{t} G_{\alpha \beta}^{i, \delta}=\left[G_{\alpha \beta}^{i, \delta}, \mathcal{H}\right]
$$

The commutator in this expression contains products of operators on different bonds $(i, \delta)$. These products are decoupled in a mean-field fashion, yielding a set of coupled linear differential equations. After a transformation to frequency and momentum space, it takes the form

$$
\omega G_{\alpha \beta}(\vec{k}, \omega)=\sum_{\alpha^{\prime} \beta^{\prime}} M_{\alpha \beta}^{\alpha^{\prime} \beta^{\prime}}(\vec{k}) G_{\alpha^{\prime} \beta^{\prime}}(\vec{k}, \omega)
$$

The dispersion relations of the collective modes are obtained from the eigenvalues of the dynamical matrix $M$, while its eigenvectors give the operators which generate these modes.

There is a problem with the above decoupling in the spinordered phases. As was discussed in Sec. IV, the low-energy fluctuations of the spin system behave differently at large $J_{F}$ and near the spin-disordering transition. For $J_{F} \ll 1$, the spins around each square plaquette are locked into a symmetric state, forming one spin-2 object, and the low-energy deformations of the spin-state are localized on the antiferromagnetic bonds. The above decoupling, which cuts across the ferromagnetic bonds, then becomes very poor. Near the spindisordering transition, the spins are rigidly coupled along the antiferromagnetic bonds and the low-energy transversal fluctuations are localized on the ferromagnetic bonds. In this case, the decoupling works well.

The crossover to spin-2 behavior at large $J_{F}$ is driven by the $\mathcal{H}_{1}$ spin-spin interaction term, Eq. (34). To avoid it, we calculate the mode spectrum of the Hamiltonian from which this term has been subtracted. The resulting dynamical matrices are listed in Appendix B. Subtracting $\mathcal{H}_{1}$ makes no difference for the spin-disordered phases, but does change the results in the AF and AFSC phase (we discuss in what way). The $\mathcal{H}_{1}$ term breaks $\mathrm{SO}(5)$ symmetry, though retaining it at the mean-field level. As a result, the model which we study in RPA has a projected $\mathrm{SO}(5)$ symmetry at the tricritical point with fine-tuned $t_{2}$.

\section{B. Mode spectrum}

We briefly discuss the mode spectrum of the antiferromagnetic and spin-disordered phases. These results are summarized in Fig. 7.

The quantum paramagnet, for which both spin and gauge symmetry are unbroken, has no Goldstone modes. Its spectrum consists of a gapped threefold degenerate spin-1 magnon mode and a pairing mode which is also gapped. The pairing gap closes at the transition to the singlet superconductor. Precisely at the transition, this mode has a quadratic dispersion. As the hole-pair density is increased from zero, it acquires a finite velocity, becoming the phase Goldstone mode of the superconducting state. This is the behavior expected at a dilute boson transition, ${ }^{32}$ of which this is a particular example (where the hole pairs are the dilute bosons). 


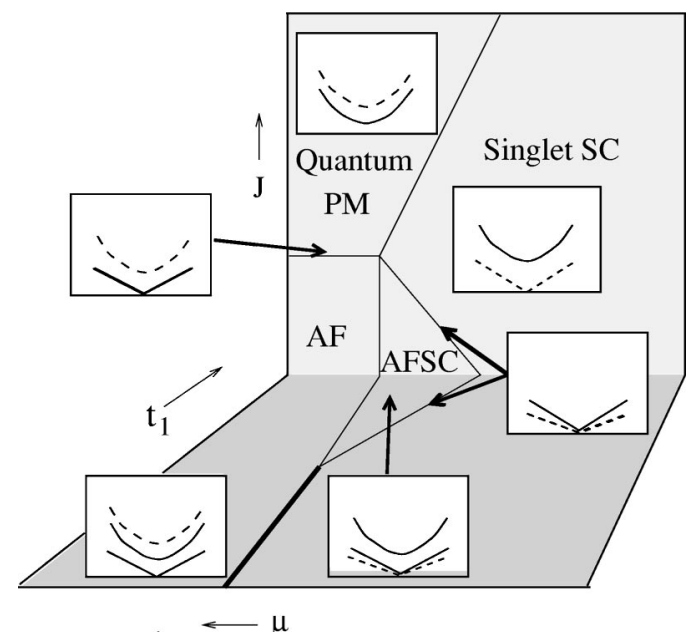

FIG. 7. Sketch of the mode spectrum in the antiferromagnetic and spin-disordered phases. The dashed lines are pairing modes when gapped (insulating phases) and phase Goldstone modes when acoustic (superconducting phases).

The threefold degenerate mode of the quantum paramagnet remains gapped through the transition to the superconductor.

The insulating antiferromagnet has a twofold degenerate spin-wave mode. In addition, it has a gapped mode related to spin-amplitude modulations and a gapped pairing mode. The spin-amplitude mode becomes degenerate with the acoustic spin-wave modes at the transition to the quantum paramagnet, where they turn into the spin-1 magnon triplet. This transition is in the same class as the spin-disordering transition of the Heisenberg bilayer model. ${ }^{33}$ The pairing gap closes at the transition to the antiferromagnetic superconductor, where it becomes the acoustic phase Goldstone mode at finite dopings. The spin-amplitude mode remains gapped through the insulator to superconductor transition, but becomes degenerate with the acoustic spin-wave modes at the subsequent transition to the singlet superconductor. At this transition line, the system therefore has four acoustic modes, of which three are degenerate.

\section{C. $\pi$ modes}

The mode spectrum as outlined above can be understood entirely from a decoupled perspective of the spin and the phase sector. At the transition line from insulator to superconductor, the pairing mode softens and continuously acquires a finite velocity. At the transition from a phase with AF order to a spin-disordered phase, the spin-amplitude mode becomes degenerate with the acoustic spin-wave modes. These two effects combined yield the described behavior, and in particular the occurrence of four acoustic modes at the AFSC to singlet SC transition. This decoupled perspective is correct for $t_{2}=0$. In this case, the gapped modes in the singlet SC state are indeed spin-1 magnons, as they are in the quantum paramagnet, while the gapped mode in the AFSC is indeed a pure spin-amplitude mode, as it is for the AF insulator.

The $t_{2}$ process, however, provides a coupling between the movement of the hole pairs and the dynamics of the spin system. This coupling changes the nature of the gapped modes in the superconducting phases with respect to those in their insulating parent phase. In the AFSC phase, the spinamplitude mode is mixed with fluctuations between the hole pair and the zero-magnetization electron-pair states. In the singlet SC phase, $\pi$ modes (hole pair to triplet fluctuations) are mixed into the spin-1 magnons.

Close to the transition from the singlet to the antiferromagnetic SC, the gapped mode becomes degenerate with the acoustic spin-wave modes and the $t_{2}$ process begins to affect the low-energy physics of the system. From the analogy with the spin-disordering transition in the insulating phase, one would expect to find a threefold degenerate acoustic magnon-mode at this transition (in addition to the phase mode). Instead, the RPA analysis yields an eigenvector

$$
\begin{aligned}
4 t_{2} \sqrt{J-2} & \left(G_{0 V}-G_{V 0}\right)+\left(J-8 t_{2}\right)\left(1-2 t_{1}+2 t_{2}\right) \\
& \times\left(G_{0 A}-G_{A 0}\right),
\end{aligned}
$$

which has both a magnon and a $\pi$-mode component. Note that this result does not change if the more natural spin-spin interactions, with $\mathcal{H}_{1}$, are used, since $\mathcal{H}_{1}$ does not affect the collective modes in the singlet $\mathrm{SC}$ phase.

For $J \rightarrow 2$, the spin-disordering line in the superconducting phase approaches half-filling. In this case, Eq. (41) becomes a pure magnon mode, which is the result expected for the insulating phase. The same eigenvector is found for $t_{2}$ $=0$, which implies that the transition in the superconducting phase is, for that case, indeed of the same type as for the insulators. The $\pi$ modes are mixed in for finite $t_{2}$. We find a pure $\pi$ mode for $t_{2}=J / 8$ and $t_{1}-t_{2}=1 / 2$. The first condition is satisfied at the point where the AFSC, triplet SC and singlet SC meet, at $n \rightarrow 0$ (see Fig. 5). This is related to the fact that the $n=0$ state and the triplet SC, which are related by a $\pi$ rotation, become at that point degenerate in energy. The second condition is of more interest: it is fulfilled if the singlet-pair hopping process and the Néel-moment interaction enter the Hamiltonian in the projected $\mathrm{SO}(5)$-symmetric form $\vec{N}_{\mathrm{p}}^{i, \delta_{1}} \cdot \vec{N}_{\mathrm{P}}^{i, \delta_{2}}$. This is of course the case at the $\mathrm{pSO}(5)$ point, which implies that the singlet SC phase at this point has the mode content expected from $\mathrm{SO}(5)$ theory: four acoustic modes, of which one is a phase mode and three are $\pi$ modes. It is shown in Ref. 15 that this is generally the case for systems with a projected $\mathrm{SO}(5)$ symmetry in the $\mathrm{SC}$ phase. The symmetry breaking due to the projection onto the lower Hubbard band shows up in the RPA mode spectrum by a different velocity for the phase and the $\pi$ modes:

$$
\begin{aligned}
\mathrm{V}_{\text {phase }}^{\mathrm{SC}, \mathrm{pSO}(5)}=\sqrt{\frac{4-J^{2}}{8}}, \\
\mathrm{~V}_{\pi}^{\mathrm{SC}, \mathrm{pSO}(5)}=\frac{2+J}{4 \sqrt{2}} .
\end{aligned}
$$

The two modes become degenerate at $J=6 / 5 \quad(n=4 / 5)$, but this point does not seem to have any special significance,

The condition $t_{1}-t_{2}=1 / 2$ can also be satisfied at the AFSC to singlet SC transition away from the point with mean field $\mathrm{SO}(5)$ symmetry. In this case, there are additional terms which break the mean field $\mathrm{SO}(5)$ symmetry, since they tune the system away from the tricritical point, but which do not affect the RPA modes. 


\section{Projected SO(5) symmetry}

In Ref. 15, the mode-spectrum at the $\operatorname{pSO}(5)$ point was studied for a general direction of the superspin. Two striking results were obtained. In the first place, the system has a twofold degenerate acoustic mode, whose velocity is independent of doping [i.e., independent of the direction of the $\mathrm{SO}(5)$-order parameter]. Secondly, the phase Goldstone mode is not acoustic, but gapless with a quadratic dispersion. It was argued that this last effect is caused by the infinite compressibility of the system at the $\operatorname{pSO}(5)$ point, where $\partial\langle n\rangle / \partial \mu$ diverges.

Both results are reproduced in our model. Since the second result is related to the infinite compressibility rather than to $\mathrm{SO}(5)$ symmetry, it always occurs at the tricritical point, also if we tune away from $t_{1}=-t_{2}=\frac{1}{4}$ while keeping $t_{1}$ $=t^{*}, \mu=\mu_{c}$. By the same argument, this effect will not disappear if the $\mathcal{H}_{1}$ term is added to the Hamiltonian, since this does not affect the mean-field phase diagram.

The first result is very sensitive to perturbations. As soon as the tricritical point is tuned away from $t_{1}=-t_{2}=\frac{1}{4}$, the velocity of the acoustic modes becomes $n$ dependent. Also the addition of $\mathcal{H}_{1}$ to the Hamiltonian destroys this effect. This can be seen by calculating the spin-wave velocity from $c=\sqrt{\rho_{s} / \chi_{\perp}}$, using the results obtained in section IV, and evaluating it at the mean-field $\mathrm{SO}(5)$ point. This yields

$$
\mathrm{v}_{s}^{\mathrm{MF} \mathrm{SO}(5)}=\sqrt{\frac{((2+J)(J+2-2 n))(J+1-n)}{8(J+2 n)}},
$$

which has an $n$ dependence. For the model without $\mathcal{H}_{1}$, the stiffness is given by [see Eqs. (21),(23)]

$$
\begin{aligned}
\rho_{s}^{\mathcal{H}-\mathcal{H}_{1}}= & \frac{2}{N \delta \phi^{2}} \Delta E(\alpha=0)=n(1-n)\left(t_{1}+t_{2}\right)(1-\cos 2 \chi) \\
& \left.+\frac{n^{2}}{4}\left(1-\cos ^{2} 2 \chi\right)\right],
\end{aligned}
$$

where $n$ and $\chi$ have the mean-field values listed in Table I. The susceptibility is obtained by multiplying the $J_{F}$ term in the mean-field energy Eq. (15) with a factor $\cos ^{2} \widetilde{\phi}^{y}$, adding the magnetic field term Eq. (16), and minimizing with respect to $\widetilde{\phi}^{y}$. We obtain

$$
\chi_{\perp}^{\mathcal{H}-\mathcal{H}_{1}}=\frac{2 n(1-\cos 2 \chi)}{J-8 t_{2}(1-n)+n(1-\cos 2 \chi)} .
$$

At the $\mathrm{pSO}(5)$ point, this stiffness and susceptibility reproduce the RPA result $\mathrm{V}_{s}=(2+J) /(4 \sqrt{2})$, which is independent of doping. Both the phase ordering and the spinordering energy contribute to the spin-wave velocity in the AFSC phase. As one approaches the $\mathrm{pSO}(5)$ point, the doping dependence of the contribution of the spin-ordering energy is precisely compensated by the opposite doping dependence of the phase-ordering contribution. Note that the model with $\mathcal{H}_{1}$ yields the same $\mathrm{v}_{s}$ at the transition to the singlet SC, where $n=(2+J) / 4$, see Eq. (43). This demonstrates the insensitivity of the RPA mode-spectrum in the singlet SC phase to the specific form of the spin-spin interactions.
At the $\mathrm{pSO}(5)$ point of our model, the acoustic modes are no longer pure spin wave, but a combination of spin wave and $\pi$ mode. Their eigenvector is given by

$$
\sqrt{1-n}\left(G_{1 V}+G_{V-1}\right)+\sqrt{n-n_{c}}\left(G_{10}+G_{0-1}\right),
$$

which starts out as a spin wave at half filling, but crosses over to a $\pi$ mode at $n=n_{c}$. This agrees with Zhang et al.'s interpretation of the doping-independent velocity in terms of the projected $\mathrm{SO}(5)$ symmetry. ${ }^{15}$

\section{SUMMARY}

We have introduced a strong coupling model for spin ordering and superconductivity. The microscopic building blocks of this model are nearest-neighbor electron pairs. The spatial structure of these pairs gives rise to $d$-wave superconductivity. At the same time, it allows the pairs to have a nonzero uniform or staggered magnetic moment. In order to avoid problems related to dimer-type spatial correlations between the pairs, the model is formulated on a 1/5-depleted lattice. A rich mean-field phase diagram is obtained, exhibiting in particular a phase which is at the same time an antiferromagnet and a superconductor. The second order lines separating this phase from the antiferromagnetic insulator and the spin-disordered superconductor end at a tricritical point, where the antiferromagnet to superconductor phase transition becomes first order. By mapping the spin sector in the antiferromagnetic phases onto a nonlinear sigma model, the main corrections to the mean-field phase diagram have been obtained.

For a specific value of one of the model parameters, a mean-field $\mathrm{SO}(5)$ symmetry between the antiferromagnetic and superconducting order-parameter appears to be realized at the tricritical point. It turns out that the model still contains spatial gradient terms which break $\mathrm{SO}(5)$ symmetry. These can be removed by modifying the spin-spin interactions. The remaining $\mathrm{SO}(5)$ symmetry breaking is then a pure quantum effect, being related to the operator algebra rather than the Hamiltonian. It is shown that true $\mathrm{SO}(5)$ symmetry can be realized for this model by allowing double site occupancy and fine-tuning the Hubbard $U$. The approximate symmetry at large $U$ is therefore a projected $\mathrm{SO}(5)$ symmetry of the kind discussed in Ref. 15.

We investigated the mode spectrum using the random phase approximation. It is found that the intersublattice hopping process gives rise to the appearance of a $\pi$ component in the gapped modes of the singlet SC phase. Approaching the point with projected $\mathrm{SO}(5)$ symmetry from the singlet SC phase, a threefold degenerate acoustic $\pi$ mode is found as well as an acoustic phase mode. The RPA mode spectrum then has the properties expected for an $\mathrm{SO}(5)$-symmetric system in the pure superconducting phase, apart from the fact that the $\pi$ modes and the phase mode have different velocities.

As reported in Ref. 15, the system at the $\mathrm{pSO}(5)$ point has a gapless phase mode with a quadratic dispersion, as well as 
a twofold degenerate acoustic mode whose velocity is independent of doping. This acoustic mode crosses over from a pure spin wave at half filling to a $\pi$ mode at the transition to the singlet SC phase. We find that the quadratic phase-mode is a property of the tricritical point rather than of the projected $\mathrm{SO}(5)$ symmetry. The doping-independent velocity, however, is a strong signature of projected $\mathrm{SO}(5)$ symmetry, which can be destroyed even by additional symmetrybreaking terms that leave the mean-field $\mathrm{SO}(5)$ symmetry intact.

\section{ACKNOWLEDGMENTS}

Financial support was provided by the Foundation of Fundamental Research on Matter (FOM), which is sponsored by the Netherlands Organization of Pure research (NWO). J.Z. acknowledges support by the Dutch Academy of Sciences (KNAW).

\section{APPENDIX A: THE SO(5) ALGEBRA}

A short overview is given of the representation of the $\mathrm{SO}$ (5) algebra for this model. In the unprojected Hilbert space, a representation of the $\mathrm{SO}(5)$ algebra can be defined which transforms the superspin $\vec{N}$ as a vector. The superspin is given by

$$
\vec{N}=(\operatorname{Re} \Delta, \overrightarrow{\widetilde{S}}, \operatorname{Im} \Delta),
$$

where

$$
\Delta^{\dagger}=\sqrt{2}\left(G_{D A}-G_{A V}\right)
$$

and $\operatorname{Re} \Delta=\frac{1}{2}\left(\Delta^{\dagger}+\Delta\right), \operatorname{Im} \Delta=1 / 2 i\left(\Delta^{\dagger}-\Delta\right)$. The generators of the $\mathrm{SO}(5)$ algebra satisfy the commutation relation

$$
\left[L_{a b}, L_{c d}\right]=i\left(\delta_{a c} L_{b d}+\delta_{b d} L_{a c}-\delta_{a d} L_{b c}-\delta_{b c} L_{a d}\right),
$$

where the indices take the values 1 through 5 . The $L_{a b}$ are antisymmetric under an interchange of $a$ and $b$. They are given by ${ }^{11}$

$$
L_{a b}=\left(\begin{array}{ccccc}
0 & & & \\
2 \operatorname{Re} \pi_{x} & 0 & & \\
2 \operatorname{Re} \pi_{y} & -S^{z} & 0 & \\
2 \operatorname{Re} \pi_{z} & S^{y} & -S^{x} & 0 \\
Q & 2 \operatorname{Im} \pi_{x} & 2 \operatorname{Im} \pi_{y} & 2 \operatorname{Im} \pi_{z} & 0
\end{array}\right)
$$

where the $\pi$ operators read $\pi_{\alpha}^{\dagger}=-\frac{1}{2} c_{1}^{\dagger} \sigma_{\alpha} \sigma_{y} c_{2}^{\dagger}$, with $\vec{\sigma}$ the Pauli matrices. ${ }^{31}$ Projecting onto the paired-electron states, we obtain

$$
\pi_{x}^{\dagger}=\frac{1}{2 i}\left(G_{D 1}-G_{D-1}+G_{1 V}-G_{-1 V}\right)
$$

$$
\begin{gathered}
\pi_{y}^{\dagger}=\frac{1}{2}\left(G_{D 1}+G_{D-1}-G_{1 V}-G_{-1 V}\right), \\
\pi_{z}^{\dagger}=\frac{i}{\sqrt{2}}\left(G_{D 0}+G_{0 V}\right) .
\end{gathered}
$$

The charge operator is given by

$$
Q=n_{D}-n_{V}
$$

It can be checked that $\vec{N}$ indeed transforms as a vector under this $\mathrm{SO}(5)$ algebra:

$$
\left[L_{a b}, N_{c}\right]=i\left(\delta_{a c} N_{b}-\delta_{b c} N_{a}\right),
$$

and furthermore that

$$
\left[N_{a}, N_{b}\right]=i L_{a b}
$$

\section{APPENDIX B: DYNAMICAL MATRICES}

A staggering factor for the antiferromagnetic spin- and the $d$-wave phase order has been absorbed into the operators $G_{\alpha \beta}$. After subtraction of $\mathcal{H}_{1}$, the Hamiltonian takes the form of a model on the square lattice, where the operators $G_{\alpha \beta}$ act on the states on the lattice sites. The Singlet dSC, $\mathrm{AF} \mathrm{dSC}$, quantum paramagnet and $\mathrm{AF}$ insulator mean-field states are all uniform in terms of these operators. It is therefore not necessary to introduce a multisublattice structure. The different modes in terms of the real (nonstaggered) operators are simply related to the ones obtained here by a shift in $k$ space.

The operators $G_{\alpha, \beta}$ separate into three sets. Each operator couples only to operators in the same set through its RPA equation of motion. One set is formed by the raising operators $\left\{G_{1 V}, G_{1 A}, G_{10}, G_{V-1}, G_{A-1}, G_{0-1}\right\}$, another by the lowering operators, which are related to this set by Hermitian conjugation. The third set contains the operators which act only on the zero-magnetization states: $\left\{G_{A V}, G_{0 V}, G_{A 0}, G_{V A}, G_{V 0}, G_{0 A}, n_{A}, n_{0}, n_{V}\right\}$.

The dynamical matrix of the raising operators has the form

$$
M_{R}=\left(\begin{array}{cc}
A^{T} & B^{T} \\
-B^{T} & -A^{T}
\end{array}\right),
$$

where $A$ and $B$ are the $3 \times 3$ matrices 


$$
\begin{gathered}
A=\left(\begin{array}{ccc}
4 \Sigma_{t} s_{\theta}^{2} \gamma_{k}+\mu-\frac{J}{4} & -2 s_{2 \theta} c_{\chi}\left(\Delta_{t}-\frac{\gamma_{k}}{4}\right) & \Sigma_{t} 2 s_{2 \theta} s_{\chi} \\
2 s_{2 \theta} c_{\chi}\left(\Sigma_{t} \gamma_{k}-\Delta_{t}\right) & c_{\theta}^{2} c_{\chi}^{2} \gamma_{k}-J & c_{\theta}^{2} s_{2 \chi} \\
2 \Sigma_{t} s_{2 \theta} s_{\chi}\left(1-\gamma_{k}\right) & \frac{1}{2} c_{\theta}^{2} s_{2 \chi}\left(2-\gamma_{k}\right) & 0
\end{array}\right), \\
B=\left(\begin{array}{ccc}
0 & \frac{1}{2} s_{2 \theta_{\theta} c_{\chi} \gamma_{k}} & 0 \\
0 & c_{\theta}^{2} c_{\chi \chi}^{2} \gamma_{k} & 0 \\
0 & -\frac{1}{2} c_{\theta}^{2} s_{2 \chi} \gamma_{k} & 0
\end{array}\right),
\end{gathered}
$$

where we have used the notation

$$
s_{x}=\sin x ; \quad c_{x}=\cos x, \quad \Sigma_{t}=t_{1}+t_{2} ; \quad \Delta_{t}=t_{1}-t_{2}, \quad \gamma_{k}=\frac{1}{2}\left[\cos \left(\vec{k} \cdot \vec{e}_{1}\right)+\cos \left(\vec{k} \cdot \overrightarrow{e_{2}}\right)\right]
$$

with $\vec{e}_{1}$ and $\vec{e}_{2}$ the basis vectors of the square lattice spanned by the long bonds, Fig. 1. The angles $\chi$ and $\theta$ are the ones appearing in the mean field energy Eq. (15). In the insulating phases, $\theta$ vanishes, while $\chi$ is equal to zero in the spin-disordered phases.

The dynamical matrix of the lowering modes is the same as $M_{R}$, apart from a minus sign. The last set has a dynamical matrix

$$
M_{0}=\left(\begin{array}{ccc}
C & D^{T} & E^{T} \\
-D^{T} & -C & -E^{T} \\
F & -F & 0
\end{array}\right),
$$

which consists of the $3 \times 3$ matrices

$$
\begin{aligned}
& C_{11}=-4 \Delta_{t}\left(c_{\chi}^{2} c_{\theta}^{2}-s_{\theta}^{2}\right) \gamma_{k}+\mu+\frac{3}{4} J, \quad C_{12}=2 \Delta_{t} c_{\theta}^{2} s_{2 \chi} \gamma_{k}-c_{\theta}^{2} s_{2 \chi}, \quad C_{13}=-2 s_{2 \theta} s_{\chi}\left(\Delta_{t} \gamma_{k}-\Sigma_{t}\right), \\
& C_{21}=2 \Sigma_{t} c_{\theta}^{2} s_{2 \chi} \gamma_{k}-c_{\theta}^{2} s_{2 \chi}, \quad C_{22}=-4 \Sigma_{t}\left(c_{\theta}^{2} s_{\chi}^{2}-s_{\theta}^{2}\right) \gamma_{k}+\mu-\frac{J}{4}, \quad C_{23}=0, \\
& C_{31}=2 \Sigma_{t} s_{2 \theta} s_{\chi}-\frac{1}{2} s_{2 \theta} s_{\chi}, \quad C_{32}=\frac{1}{2} s_{2 \theta} c_{\chi} \gamma_{k}, \quad C_{33}=-c_{\theta}^{2} c_{2 \chi} \gamma_{k}+J \\
& D=\left(\begin{array}{ccc}
0 & 0 & \frac{1}{2} s_{2 \theta{ }{ }_{\chi}} \gamma_{k} \\
0 & 0 & 2 \Delta_{t} s_{2 \theta} c_{\chi}-\frac{1}{2} s_{2 \theta} c_{\chi} \gamma_{k} \\
0 & -2 s_{2 \theta} c_{\chi}\left(\Delta_{t}-\Sigma_{t} \gamma_{k}\right) & c_{\theta}^{2} c_{2 \gamma} \gamma_{k}
\end{array}\right) \\
& E=\left(\begin{array}{ccc}
-2 \Delta_{t} s_{2 \theta} c_{\chi}\left(1-\gamma_{k}\right) & 0 & c_{\theta}^{2} s_{2 \chi} \\
0 & 2 \Sigma_{t} s_{2 \theta_{\theta} s_{\chi}}\left(1-\gamma_{k}\right) & -c_{\theta}^{2} s_{2 \chi} \\
2 \Delta_{t} s_{2 \theta} c_{\chi}\left(1-\gamma_{k}\right) & -2 \Sigma_{t} s_{2 \theta} s_{\chi}\left(1-\gamma_{k}\right) & 0
\end{array}\right) \text {, } \\
& F=\left(\begin{array}{ccc}
-2 \Delta_{t} s_{2 \theta} c_{\chi} & 0 & c_{\theta}^{2} s_{2 \chi} \\
0 & 2 \Sigma_{t} s_{2 \theta} s_{\chi} & -c_{\theta}^{2} s_{2 \chi} \\
2 \Delta_{t} s_{2 \theta} c_{\chi} & -2 \Sigma_{t} s_{2 \theta s_{\chi}} & 0
\end{array}\right)
\end{aligned}
$$

For the singlet SC phase $(\chi=0)$, the operators $G_{\alpha \beta}$ with $\alpha$ and $\beta$ referring to triplet states decouple from the equations of motion, since there is no longer a triplet component in the mean-field ground state. This leaves the raising set $\left\{G_{1 V}, G_{1 A}, G_{V-1}, G_{A-1}\right\}$ and the Hermitian conjugate lowering set. The set of zero-magnetization operators splits into the 
transversal set $\left\{G_{0 V}, G_{0 A}, G_{V 0}, G_{A 0}\right\}$ and the longitudinal set $\left\{G_{A V}, G_{V A}, n_{A}-n_{V}\right\}$. The dynamical matrices of the first three sets contain the threefold degenerate $\pi$ - and spin-1 magnon modes. The fourth set contains the phase and the pairing mode.

In the quantum paramagnet phase $(\chi=\theta=0)$, the first three sets further simplify. Since the $\pi$ operators do not refer to the spin-singlet ground state, only the spin-1/charge-0 operators are left in these sets. The phase-mode $n_{A}-n_{V}$ disappears from the last set.

${ }^{1}$ P. Nozieres and S. Schmitt-Rink, J. Low Temp. Phys. 59, 159 (1985)

${ }^{2}$ J. R. Engelbrecht, A. Nazarenko, M. Randeria, and E. Dagotto, Phys. Rev. B 57, 3406 (1998).

${ }^{3}$ Ch. Renner, B. Revaz, K. Kadowaki, M. Aprile, and O. Fischer, Phys. Rev. Lett. 80, 3606 (1998).

${ }^{4}$ V. J. Emery and S. Kivelson, Nature (London) 374, 434 (1995).

${ }^{5}$ D. Pines cond-mat/9702187 (unpublished).

${ }^{6}$ J. M. Tranquada, B. J. Sternlieb, J. D. Axe, Y. Nakamura, and S. Uchida, Nature (London) 375, 561 (1995).

${ }^{7}$ G. M. Luke, K. Kojima, M. Larkin, J. Merrin, B. Nachumi, Y. J. Uemura, Y. Nakamura, S. Uchida, and M. Crawford, Hyperfine Interact. 105, 113 (1997); W. Wagener, H.-H. Klauß, M. Hillberg, M. A. C. de Mello, M. Birke, F. J. Litterst, B. Büchner, and H. Micklitz, Phys. Rev. B 55, R14 761 (1997).

${ }^{8}$ H. Kimura, K. Hirota, H. Matsuhita, K. Yamada, Y. Endoh, S.-H. Lee, Ch. F. Majkrzak, R. Erwin, G. Shirane, M. Greven, Y. S. Lee, M. A. Kastner, and R. J. Birgeneau, Phys. Rev. B 59, 6517 (1999)

${ }^{9}$ J. M. Tranquada, J. D. Axe, N. Ichikawa, A. R. Moodenbaugh, Y. Nakamura, and S. Uchida, Phys. Rev. Lett. 78, 338 (1997).

${ }^{10}$ A. H. Castro-Neto and D. Hone, Phys. Rev. Lett. 76, 2165 (1996); C. N. A. van Duin and J. Zaanen, ibid. 80, 1513 (1998); S. Sachdev, Phys. World 12, 33 (1999).

${ }^{11}$ S. C. Zhang, Science 275, 1089 (1997).

${ }^{12}$ J. Zaanen, Physica C 318, 217 (1999).

${ }^{13}$ M. Vojta and S. Sachdev, Phys. Rev. Lett. 83, 3916 (1999).

${ }^{14}$ R. Eder, A. Dorneich, M. G. Zacher, W. Hanke, S.-C. Zhang, Phys. Rev. B 59, 561 (1999).

${ }^{15}$ S.-C. Zhang, J.-P. Hu, E. Arrigoni, W. Hanke, and A. Auerbach, Phys. Rev. B 60, 13070 (1999).

${ }^{16}$ G. Sierra, M. A. Martin-Delgado, J. Dukelsky, and S. R. White,
Phys. Rev. B 57, 11666 (1998); M. Troyer, H. Tsuretsugu, and T. M. Rice, ibid. 53, 251 (1996).

${ }^{17}$ D. Rokhsar and S. Kivelson, Phys. Rev. Lett. 61, 2376 (1988).

${ }^{18}$ E. Fradkin and S. Kivelson, Mod. Phys. Lett. B 4, 225 (1990).

${ }^{19}$ P. W. Kasteleyn, Physica (Amsterdam) 27, 1209 (1961).

${ }^{20}$ R. Micnas, J. Ranninger, and S. Robaszkiewicz, Rev. Mod. Phys. 62, 113 (1990).

${ }^{21}$ C. N. A. van Duin and J. Zaanen, Phys. Rev. Lett. 78, 3019 (1997).

${ }^{22}$ A. van Otterlo, K.-H. Wagenblast, R. Baltin, C. Bruder, R. Fazio, and G. Schön, Phys. Rev. B 52, 16176 (1995).

${ }^{23}$ F.D.M. Haldane, Phys. Lett. 93A, 464 (1983); Phys. Rev. Lett. 50, 1153 (1983)

${ }^{24}$ S. Chakravarty, B. I. Halperin, and D. R. Nelson, Phys. Rev. Lett. 60, 1057 (1988); Phys. Rev. B 39, 2344 (1989).

${ }^{25}$ J. Igarahsi, Phys. Rev. B 46, 10763 (1992).

${ }^{26}$ G. Aeppli, T. E. Mason, S. M. Hayden, H. A. Mook, and J. Kulda, Science 278, 1432 (1997).

${ }^{27}$ V. Barzykin and D. Pines, Phys. Rev. B 52, 13585 (1995); Y. Zha, V. Barzykin, and D. Pines, cond-mat/9601016 (unpublished).

${ }^{28}$ A.V. Chubukov, S. Sachdev, and J. Ye, Phys. Rev. B 49, 11919 (1994).

${ }^{29}$ E. W. Carlson, S. A. Kivelson, V. J. Emery, and E. Manousakis, Phys. Rev. Lett. 83, 612 (1999).

${ }^{30}$ S. C. Zhang, cond-mat/9808309 (unpublished).

${ }^{31}$ D. Scalapino, S. C. Zhang, and W. Hanke, Phys. Rev. B 58, 443 (1998).

${ }^{32}$ S. Sachdev, Quantum Phase Transitions (Cambridge University Press, New York, 1999).

${ }^{33}$ A. V. Chubukov and D. K. Morr, Phys. Rev. B 52, 3521 (1995).

${ }^{34}$ S.B. Haley and P. Erdös, Phys. Rev. B 5, 1106 (1972). 